\title{
Poster: Automatic Personal Fitness Assistance through Wearable Mobile Devices
}

\author{
Xiaonan Guo, Jian Liu, Yingying Chen \\ Stevens Institute of Technology, Hoboken, NJ 07030, USA \\ \{xguo6, jliu28, yingying.chen\}@stevens.edu
}

\begin{abstract}
Acknowledging the powerful sensors on wearable mobile devices enabling various applications to improve users' life styles and qualities, this paper takes one step forward developing a automatic personal fitness assistance through wearable mobile devices to assess dynamic postures in workouts. In particular, our system recognizes different types of exercises and interprets fine-grained fitness data to an easy-to-understand exercise review score. The system has the ability to align the sensor readings from wearable devices to the earth coordinate system, ensuring the accuracy and robustness of the system. Experiments with 12 types of exercises involve multiple participants doing both anaerobic and aerobic exercises in indoors as well as outdoors. Our results demonstrate that the proposed system can provide meaningful review and recommendations to users by accurately measure their workout performance and achieve $93 \%$ accuracy for workout analysis.
\end{abstract}

\section{INTRODUCTION}

The proliferation of wearable mobile devices (e.g., smartwatches, fitness bands, and smartphones mounted on arms) has already shown its potential on improving our life styles. An important use case of wearable mobile devices is providing guidelines to improve people's daily activities, for example, tracking walking steps, monitoring sleep qualities, and estimating daily caloric intake.

In this work, we take one step forward by answering the question: Whether such wearable mobile devices become powerful enough leveraging fine-grained sensing information to perform systematic comprehensive fitness assistance.

Traditionally, fitness monitoring is performed by analyzing the workout captured by video tapes [1] or specialized sensors [2]. In recent years, fitness trackers and dedicated devices, such as Fitbit [3] and Gym watch [4], show the initial success of fitness monitoring. However, Gym watch requires people to purchase dedicated sensors and wear them during exercises.

Toward this end, we propose a personal fitness assistant leveraging wearable mobile devices to achieve the following two main aspects: (i) Fine-grained Fitness Data Interpretation. Recording the sensor readings on wearable mobile devices (e.g., smart-

Permission to make digital or hard copies of part or all of this work for personal or classroom use is granted without fee provided that copies are not made or distributed for profit or commercial advantage and that copies bear this notice and the full citation on the first page. Copyrights for third-party components of this work must be honored. For all other uses, contact the owner/author(s).

MobiCom'16 October 03-07, 2016, New York City, NY, USA

(C) 2016 Copyright held by the owner/author(s).

ACM ISBN 978-1-4503-4226-1/16/10.

DOI: http://dx.doi.org/10.1145/2973750.2985266

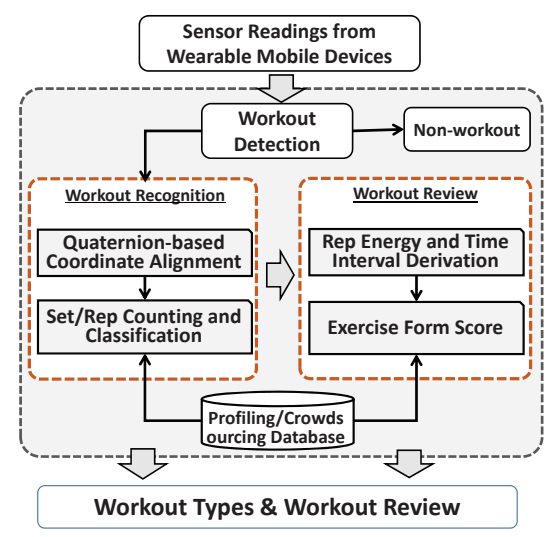

Figure 1: System framework.

watches) during workout to explore their capability of deriving finegrained exercise information including exercise types, the number of set and the number of repetitions (reps) per set. (ii) Smart Exercise Guidance. The proposed system aims to not only regulate the workouts by following the Frequency, Intensity, Time and Type (FITT) principle [5], but also provide detailed guidelines to review the user's posture and provide recommendation in keeping correct exercise form.

In particular, the system exploits Short Time Energy (STE) to derive fine-grained fitness data (i.e., strength and speed of body movements) in exercises and recognizes different types of exercises automatically by using embedded sensors on wearable mobile devices. We develop a novel metric for evaluating the quality of each user's exercises, exercise form score. This exercise form score reflects the difference of strength and speed of body movements between each repetition of an exercise based on a reference profile. The reference profile could be either obtained from the user's own sensor data or built from other people's data (e.g., training coaches or members from the same fitness club) through crowdsourcing platforms (e.g., fitness club's facebook, WhatsApp or WeChat).

\section{SYSTEM DESIGN}

\subsection{System Overview}

As illustrated in Figure 1, the system takes as input time-series of sensor readings including acceleration and quaternion, all of which are readily available in off-the-shelf wearable mobile devices. Our system first performs Workout Detection to filter out non-workout activities. Then, the system provides fine-grained workout types and workout reviews based on Workout Recognition and Work Review. 


\subsection{Workout Recognition}

Workout Detection. A key observation is that most regular exercises involve repetitive arm movements and non-workout activities usually don't have such long-term repetitive pattern. Then, we adopt an autocorrelation-based approach. Particularly, we use the Magnitude of Linear Acceleration $(M L A)$ to estimate the linear acceleration of exercise motions. Then we calculate the autocorrelation of the time series of MLA and find the number of peaks in the autocorrelation. Finally, we use a threshold-based method to confirm the detected repetitive patterns are resulted from workouts.

Quaternion-based Coordinate Alignment. The orientation of wearable mobile devices may change during exercises due to rotation caused by arm movement. Therefore, to ensure the same exercise exhibits unique sensor reading pattern, we need to convert the sensor readings from the mobile device coordinate $p_{m}$ to a fix coordinate system, i.e., the earth coordinate $p_{e}$. In particular, we use the quaternion-based rotation $p_{e}=q_{m e} p_{m} q_{m e}^{-1}$, where $q_{m e}$ is the quaternion reading from the mobile device coordinate to the earth coordinate, which can be obtained from the device directly. $q_{m e}^{-1}$ is the conjugate quaternion of $q_{m e}$.

Set/Rep Counting and Classification. We adopt the Short Time Energy (STE) to capture the unique energy pattern in the time series of MLA. The system finds the local minimum point between two peaks as the ending point of each repetition, and the data between two detected ending point are defined as a segment of repetition.

Then, we extract nine statistical acceleration-based features and utilize a light-weight SVM-based approach for classification. It is light-weight because the system only needs to determine the workout type for the first few rep segments within a set, and the workout type is identified as the majority decision.

\subsection{Workout Review}

Our system aims to offer users a more intuitive way to understand their performance in exercises by comparing their exercise statistics to a baseline, which could be either generated based on the users' own data or based on the data from croudsourcing. The metric was based on rep energy and time interval of each rep.

Rep Energy and Time Interval Derivation. We utilize the energy level of each rep to describe the Motion Strength (MS), which means a set of reps with good performance should maintain a stable energy level. The energy level of each rep can be estimated by the maximum value in obtained STE of MLA.

Furthermore, we utilize the time period of each rep to describe the Performing Period $(P P)$ of each rep and good performance should also have similar time periods.

Exercise Form Score. Based on these two criteria, we define the Exercise Form Score including two subscores: $M S$ score and $P P$ score. The subscores depicts how the testing rep deviates from the baseline. Particularly, the MS score for the $i^{\text {th }}$ rep is defined as:

$$
E_{i}=\frac{A(i)-A^{*}}{A^{*}}, \quad i=1,2,3, \ldots, n,
$$

where $A(i)$ is the maximum STE of the MLA of the $i^{\text {th }}$ rep, and $A^{*}$ is the motion strength baseline. Similarly, the PP score for the $i^{\text {th }}$ rep is defined as

$$
T_{i}=\frac{I(i)-I^{*}}{I^{*}}, \quad i=1,3,4, \ldots, n,
$$

where $I_{i}$ is the length of the $i^{t h}$ rep and $I^{*}$ is the performing period baseline. The output exercise form score is a 2-tuple score that can be denoted as $\left\langle E_{i}, T_{i}\right\rangle$.

We design two baselines that are suitable in different scenarios,

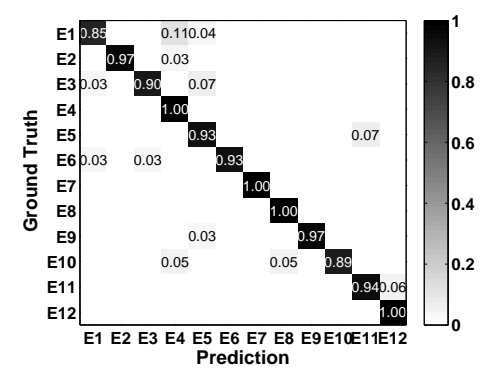

Figure 2: Performance of recognizing 12 exercises by using smartwatches.

namely Personal Baseline and Crowdsouring Baseline. (1) Personal Baseline. We observe that users usually can perform exercises with standard strength and frequency at the beginning of the workout. Based on this observation, a good candidate of the baseline is the early portion of the user's own reps. In particular, we derive the personal baseline by averaging the motion strength and performing period of the first $k$ reps of the first set in the user's sensor data. We empirically choose $k=5$ in our work. (2) Crowdsourcing Baseline. The personal baseline is good for short-term exercise performance evaluation but could be bias to the user's own preference. To tackle this problem, we further propose the crowdsourcing baseline, which allows users to compare their performance with the baseline from exemplars (e.g., fitness coaches, bodybuilders, and amateur expertise) to achieve a long-term and more accurate exercise performance evaluation.

\section{PRELIMINARY EVALUATION}

We evaluate our system with a smartwatch of LG Watch Urbane. In our experiments, all volunteers are asked to wear the smartwatch with their own wearing preference. (i.e., different device facing directions) We test on 12 different exercises and collect acceleration and quaternion with the sampling rate of $100 \mathrm{~Hz}$. The ground truth of workout statistics are recorded by a volunteer.

Figure 2 shows the confusion matrix of the recognizing exercise types by using smartwatches. In the confusion matrix, an entry $M_{i j}$ denotes the percentage between the number of exercise $i$ was predicted as gesture $j$ and the total number of $i$. We observe that the average accuracy for smartwatch is $95 \%$ with standard deviation $5 \%$ over all 12 types of exercises. The above results show that our system can automatically provide fine-grained exercise type recognition by using wearable mobile devices without user involvement.

\section{ACKNOWLEDGMENTS.}

This work is supported in part by the NSF grants CNS1409767 and CNS1514436.

\section{REFERENCES}

[1] Juliane Arney. You should be in pictures! experts share tips on how to produce a quality fitness video for any purpose. IDEA Fitness Journal, 2(1):86-90, 2005.

[2] Keng-hao Chang and et al. Tracking free-weight exercises. In ACM UbiComp, 2007.

[3] Fitbit. http://www.fitbit.com/, 2014.

[4] Gymwatch. https://www.gymwatch.com/.

[5] American College of Sports Medicine et al. ACSM's guidelines for exercise testing and prescription. Lippincott Williams \& Wilkins, 2013. 